Original paper

\title{
Characterisation of a high resolution small field of view portable gamma camera
}

\author{
S.L. Bugby ${ }^{\mathrm{a}, *}$, J.E. Lees ${ }^{\mathrm{a}}$, B.S. Bhatia ${ }^{\mathrm{a}, \mathrm{b}}$, A.C. Perkins ${ }^{\mathrm{c}}$ \\ a Space Research Centre, University of Leicester, Leicester LE1 7RH, UK \\ ${ }^{\mathrm{b}}$ Clinical Imaging Group, Sandwell and West Birmingham NHS Hospital Trust, West Bromwich B71 4HJ, UK \\ ${ }^{\mathrm{c}}$ Radiological and Imaging Sciences, School of Medicine, University of Nottingham, Nottingham NG7 2UH, UK
}

\section{A R T I C L E I N F O}

\section{Article history:}

Received 3 June 2013

Received in revised form

27 September 2013

Accepted 21 October 2013

Available online 10 November 2013

\section{Keywords:}

Small field of view gamma camera

Gamma camera performance

Gamma camera specification

Gamma camera characteristics

\begin{abstract}
A B S T R A C T
A handheld, high-resolution small field of view (SFOV) pinhole gamma camera has been characterised using a new set of protocols adapted from standards previously developed for large field of view (LFOV) systems. Parameters investigated include intrinsic and extrinsic spatial resolution, spatial linearity, uniformity, sensitivity, count rate capability and energy resolution. Camera characteristics are compared to some clinical LFOV gamma cameras and also to other SFOV cameras in development.
\end{abstract}

(c) 2013 Associazione Italiana di Fisica Medica. Published by Elsevier Ltd. All rights reserved.

\section{Introduction}

Nuclear medical images are typically acquired using large field of view (LFOV) gamma cameras designed for whole body scanning and SPECT imaging. A new generation of small field of view (SFOV) gamma cameras, now in development, has been designed to provide higher resolution capabilities for specific procedures, such as sentinel node localisation [1].

Standardised procedures for assessing the performance characteristics of medical gamma cameras have generally been based on the original standards published by the US National Electrical Manufacturing Association (NEMA) [2]. In the UK and Europe a comprehensive description of procedures to be carried out in clinical departments has been developed by the Institute of Physics and Engineering in Medicine (IPEM) [3]. However, these tests are designed for use with standard LFOV gamma cameras and are not necessarily applicable to SFOV systems. The term SFOV itself is ambiguous, and has been used for cameras with FOVs between $40 \times 40 \mathrm{~cm}$ and $40 \times 40 \mathrm{~mm}$. For cameras working towards the higher end of this range, the IPEM standards are often appropriate. For those instruments operating towards the lower end, these

\footnotetext{
* Corresponding author. Tel.: +44 1162297720 .

E-mail address: s.bugby@le.ac.uk (S.L. Bugby).
}

standards may not be applicable and, in some cases, the procedures may even be impossible to perform.

In this communication the performance of a SFOV gamma camera currently in development is fully characterised following protocols developed specifically for use with SFOV gamma cameras. Performance characteristics are then compared to those of several standard LFOV systems currently in clinical use, and to similar SFOV cameras in development.

\section{Materials}

The portable compact gamma camera (CGC) has been developed by the Space Research Centre, University of Leicester in collaboration with Radiological and Imaging Sciences at the University of Nottingham. The current camera incorporates a number of improvements on the design previously described in the literature [4], particularly in terms of improved shielding and a new cooling system.

The CGC consists of a $0.5 \mathrm{~mm}$ diameter pinhole with an acceptance angle of $60^{\circ}$ in a $6 \mathrm{~mm}$ thick tungsten collimator, with a detector placed at a distance of $10 \mathrm{~mm}$ from the pinhole centre. An interchangeable collimator is separated from the detector system by a $1 \mathrm{~mm}$ thick Al window for protection. The detector is a $0.5 \mathrm{~mm}$ thick Tl doped CsI scintillator, consisting of multiple closely packed $\operatorname{CsI}(\mathrm{Tl})$ columns each a few $\mu \mathrm{m}$ wide, joined to an electron multiplying charge coupled device (EMCCD) with Dow Corning optical grease. The EMCCD used is the back-illuminated CCD97 
produced by e2v technologies [5]. The active imaging area of the EMCCD measures $\sim 8 \times 8 \mathrm{~mm}$ but, due to the use of a pinhole collimator, the field of view of the CGC can be larger than this depending upon the magnification factor (related to imaging distance). The detector is Peltier-cooled to temperatures of between 0 and $-14{ }^{\circ} \mathrm{C}$. Tungsten shielding $3 \mathrm{~mm}$ thick surrounds the detector enclosure. A photograph and schematic of the CGC can be seen in Fig. 1.

Individual photon events are detected using automatic scale selection ('blob detection') [4]. Bespoke analysis software fits a Gaussian distribution to each light splash recorded on the detector. Each fitted Gaussian equates to a single gamma photon event in the scintillator. The peak amplitude and standard deviation of the Gaussian may be used to calculate the energy of the interacting gamma photon. A new image can then be created, either from recreated light splashes based on the Gaussian information or from the centre points of individual gamma events.

\section{Performance specification}

Unless otherwise stated, all images in this communication underwent an image correction process as follows. Hot pixels, defined as those recording counts above expected thermal noise in more than $5 \%$ of frames in a dark image, were replaced with the average signal value of their 4 nearest neighbours. A flood image was taken, either with a point source at a large distance (for intrinsic measurements) or with a uniform flood source (extrinsic measurements), regularly throughout each day of testing. Dark images, with no incident illumination, were taken regularly during experimentation. The dark and flood images were first corrected for hot pixels. A master flat image was then created by subtracting the dark image from the flood image (corrected for any difference in exposure time) and then normalising the resulting image to its maximum value. After hot pixel removal an image would be corrected for flat field effects by subtracting the dark image and then dividing by the master flat image.

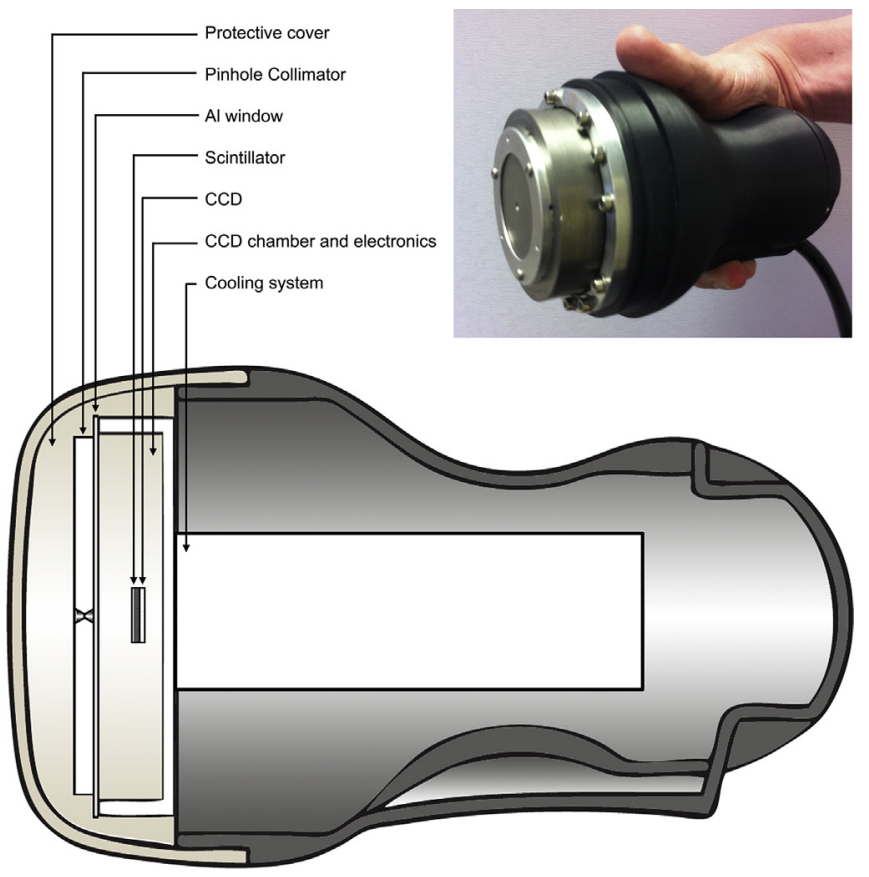

Figure 1. Main: schematic of compact gamma camera (CGC). Inset: image of CGC with protective cover removed to show pinhole collimator.

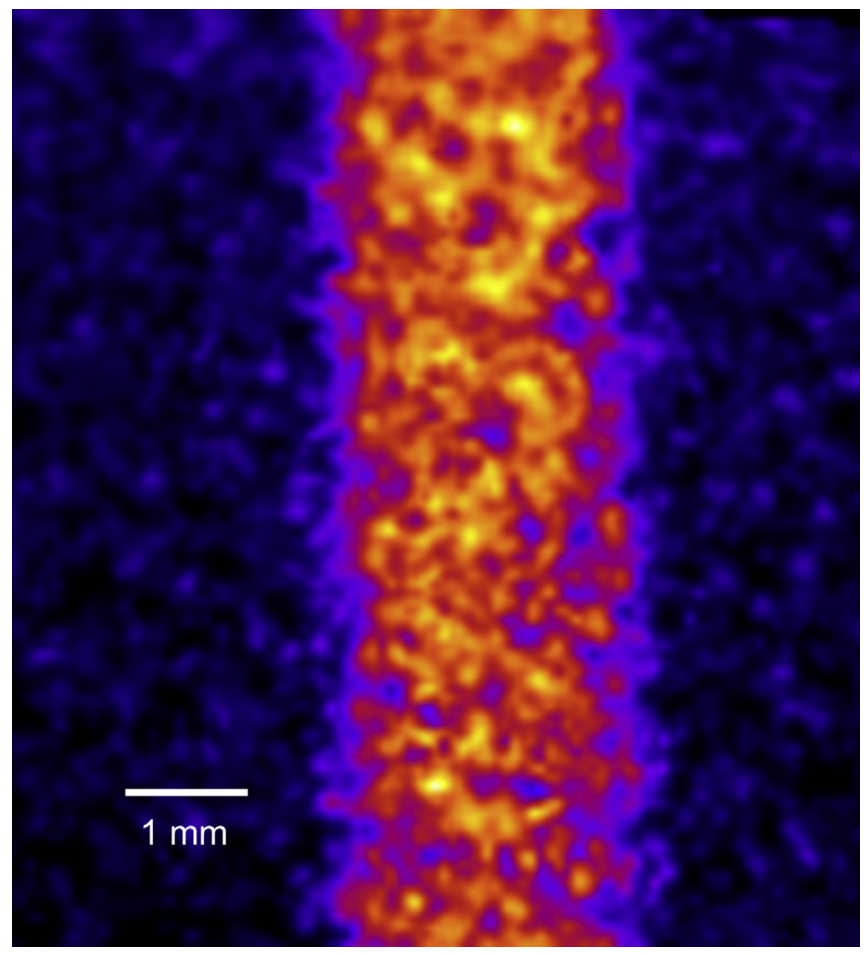

Figure 2. Example slit image produced for intrinsic spatial resolution calculations Image is of a $3 \mathrm{~mm}$ diameter point source at a distance of $250 \mathrm{~mm}$ from the detector masked by a $2 \mathrm{~mm}$ wide transmission slit phantom at a distance of $50 \mathrm{~mm}$ from the detector. Image was taken for 35 min, 10,000 frames. Peak counts per pixel are 480 .

$4 \times 4$ pixel binning was used in all cases, producing a $104 \times 112$ pixel usable image array with pixel sizes of $64 \times 64 \mu \mathrm{m}$. Binning was used as individual CCD pixels $(16 \mu \mathrm{m})$ would grossly oversample for expected resolutions of the order of several hundred $\mu \mathrm{m}$. Edge pixels were removed to eliminate the effect of defects at the machined edge of the scintillator. Raw data were converted to gamma photon counted images using the blob detection method.

\section{Intrinsic spatial resolution}

Intrinsic spatial resolution was calculated using an edge response function (ERF) method. A $10 \mathrm{~mm}$ thick lead block, with a $2 \times 20 \mathrm{~mm}$ slit, was positioned $40 \mathrm{~mm}$ in front of the uncollimated camera face ( $50 \mathrm{~mm}$ from the detector). A $3 \mathrm{~mm}$ diameter $14 \mathrm{MBq}$ ${ }^{99 \mathrm{~m}} \mathrm{Tc}$ source was placed $200 \mathrm{~mm}$ above the slit. At this height it can be assumed that the photons from the source impinge perpendicular to the slit and detector and are parallel to one another. A 10,000 frame image was taken (35 min acquisition time) with the CGC, an example of which is shown in Fig. 2. An additional image was taken with a $10 \mathrm{~mm}$ thickness of Perspex placed between the slit and the camera as a scattering medium. Peak pixel counts were 480 and 390 respectively.

In some instances the slit collimator was not perfectly orientated parallel to the detector array's principal axes. This was accounted for by a least squares fitting algorithm which calculated the centre line of the slit image and its angle to detector array axes. The ERF (the relative intensity compared to distance from edge) was then calculated.

Although it would be possible to use the ERF as a measure of resolution, the IPEM standard recommends the full width half maximum (FWHM) of a line spread function (LSF). The full width tenth maximum (FWTM) is also reported as it is not expected for the LSF to be Gaussian. The LSF was calculated simply as the 
derivative of the ERF. Figure 3 shows the ERF and LSF for the example image in Fig. 2. This analysis was completed for each edge.

The modulation transfer function (MTF) was also calculated by performing a Fast Fourier Transform of the LSF. This describes the camera response in frequency space. An example MTF is shown in
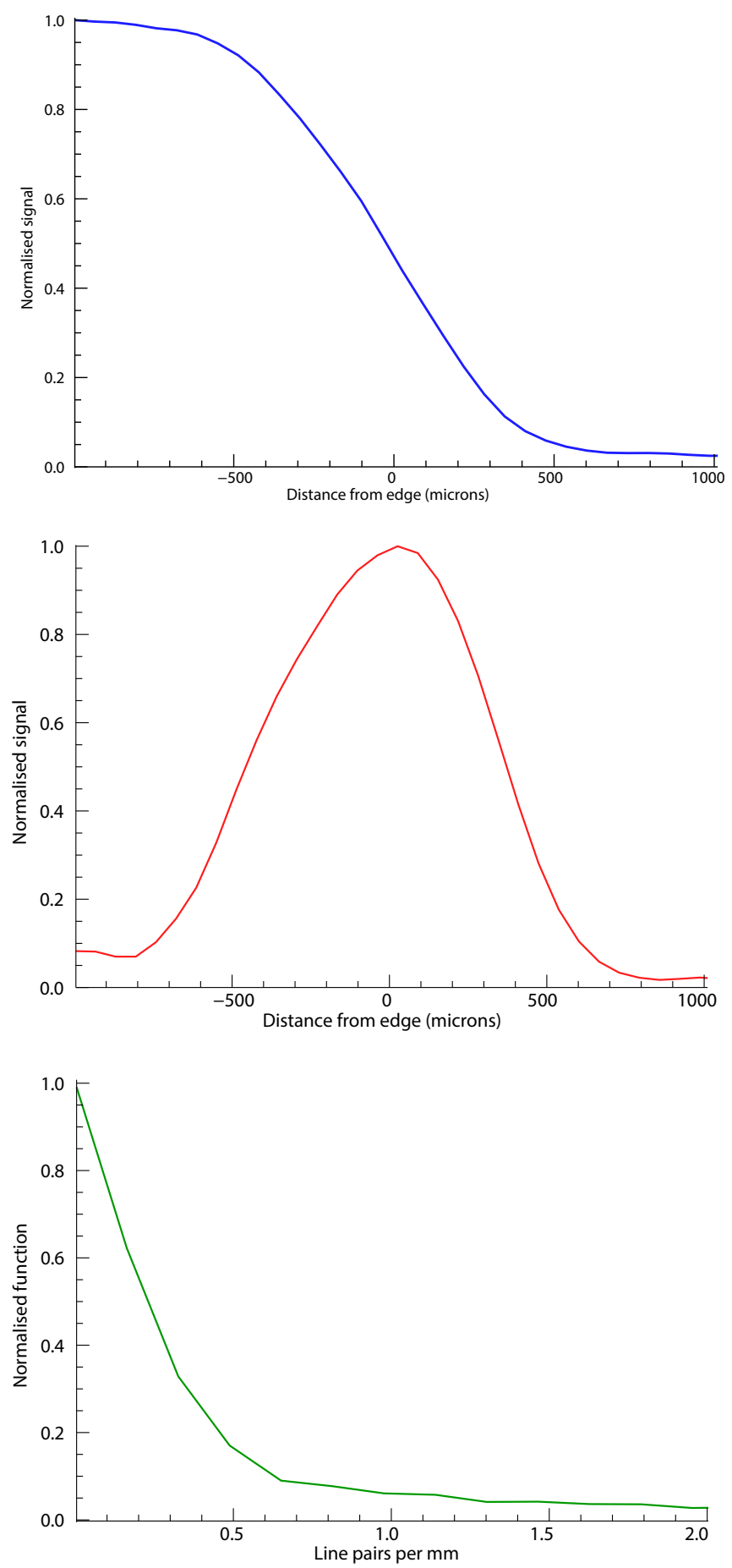

Figure 3. Graphs showing the intrinsic resolution calculation process for the right hand edge of Fig. 2. Top: Edge response function (ERF). The edge is taken to be $1 \mathrm{~mm}$ from the fitted centre of the slit image. Centre: Line spread function (LSF) calculated as the derivative of the ERF. Asymmetry may indicate that the width of the slit was not sufficient to behave as two distinct, independent edges. Bottom: Modulation transfer function (MTF), the fast Fourier transform of the LSF. The profile shows the best response at low frequencies and falls off quickly as frequency increases. the bottom graph of Fig. 3. The MTF shows the best response at low frequencies, as would be expected, and drops quickly as frequency increases. Bar patterns cease to be visible at around the $10 \%$ point of the MTF, so this is the value typically quoted, although occasionally $5 \%$ or $3 \%$ values are given instead.

Table 1 shows a range of typical resolution measurements, using an average of both edges of the slit, for slits orientated vertically and horizontally across the detector face. General trends are as expected, with resolutions degrading with increasing Perspex thickness.

\section{System spatial resolution}

It has long been established that the spatial resolution using a pinhole collimator will vary based on the distance between the pinhole and the source [6]. The nature of a pinhole collimator makes measurement of spatial resolution at the collimator face (as would be applicable for a parallel hole collimator) unhelpful for clinical assessment. Resolution measurements were taken at a range of distances, using a $0.5 \mathrm{~mm}$ diameter pinhole, and used to calculate a relationship that may be applied to any situation.

A $1 \mathrm{~mm}$ diameter capillary tube filled with $40 \mathrm{MBq}$ of ${ }^{99 \mathrm{~m}} \mathrm{Tc}$ was used as a line source. Varying numbers of $4 \mathrm{~mm}$ thick blocks of Perspex were placed directly in front of the camera face. The capillary was then positioned directly in front of the Perspex blocks. In this way the capillary was imaged at a range of distances from the camera with Perspex filling the intervening space.

The resolution of a pinhole camera will vary across its field of view, so all phantom images were taken within the $30^{\circ}$ FOV of the pinhole where this effect is negligible [7]. Image acquisition time was $100 \mathrm{~s}$. The capillary tube was orientated at a range of angles to the detector array and this orientation was corrected for as described in Section 3.1.

For each image the profile of the capillary tube was taken and FWHM and FWTM values calculated. These values were then corrected for magnification to give the resolution of the camera in terms of the object it was seeing rather than the image it was producing. A plot of the calculated resolutions against Perspex thickness can be seen in Fig. 4.

Least squares fitting produces a linear fit with $R^{2}=0.998$ for the FWHM data and $R^{2}=0.998$ for the FWTM data. As would be expected, resolutions degrade with increasing depths of Perspex.

FWTM values increase faster than FWHM values due to the decrease in signal to noise ratio with increasing thickness of Perspex. At greater thicknesses, unscattered counts are reduced and scattered counts act to enhance the background noise level. The overall effect is larger on FWTM values where signal is already relatively low.

For LFOV cameras resolution is stated as a single value, measured at the collimator face. Clearly this would not be a practical measurement in this case. The FWHM resolution at the non-magnifying position (13 $\mathrm{mm}$ ) was found to be $1.28 \mathrm{~mm}$, and this varies according to the linear relationship between distance, $d$ (with scattering medium), and resolution. For the CGC this relationship is:

FWHM $=0.0432 d+0.727$

FWHM calculated with Equation (1) is of the order of the geometric resolution of a pinhole with the discrepancy coming from scatter, leakage through the collimating material and photon spreading within the scintillator. Equation (1) only holds for values of $d$ greater than the non-magnifying distance.

\section{Spatial linearity}

Slit collimated images were taken and analysed as described in Section 3.1. 
Table 1

CGC intrinsic resolution measurements.

\begin{tabular}{lll}
\hline & \multicolumn{2}{l}{ Perspex thickness $(\mathrm{mm})$} \\
\cline { 2 - 3 } & 0 & 10 \\
\hline ERF 90\%-10\% $(\mu \mathrm{m})$ & 811 & 841 \\
LSF FWHM $(\mu \mathrm{m})$ & 634 & 743 \\
LSF FWTM $(\mu \mathrm{m})$ & 1061 & 1128 \\
MTF 10\% $($ line pairs per $\mathrm{mm})$ & 0.92 & 0.83 \\
MTF 10\% $(\mu \mathrm{m})$ & 545 & 600 \\
\hline
\end{tabular}

Slit centre positions for each row were compared to the least squares calculated centre positions to calculate the deviation statistics outlined in Table 2. Rows where a centre position could not be found due to low signal to noise ratios were excluded from this analysis (87/113 rows remaining). As the true orientation of the collimator is not determined directly this method will only find random errors in linearity, not systematic ones.

\section{Intrinsic spatial uniformity}

There are a number of quantitative measures of spatial uniformity used in the quality control of medical gamma cameras. Standard measurements include the coefficient of deviation (the ratio of standard deviation in counts to mean counts quoted as a percentage), integral uniformity and differential uniformity. IPEM Report 86 [3] suggests that at least one integral and one differential value should be quoted with preference given to the coefficient of variation and the spread of differential uniformity as the most effective methods. The integral uniformity (IU) can also be quoted, which can be calculated using Equation (2), where $C$ is the number of counts per pixel.

$\mathrm{IU}=\frac{C_{\max }-C_{\min }}{C_{\max }+C_{\min }} \times 100 \%$

These measures give an idea of global uniformity but do not look at local variations. Differential uniformity (DU) can be calculated by using Equation (2) for only a localised number of pixels. IPEM standards suggest calculating differential uniformity 10 times for

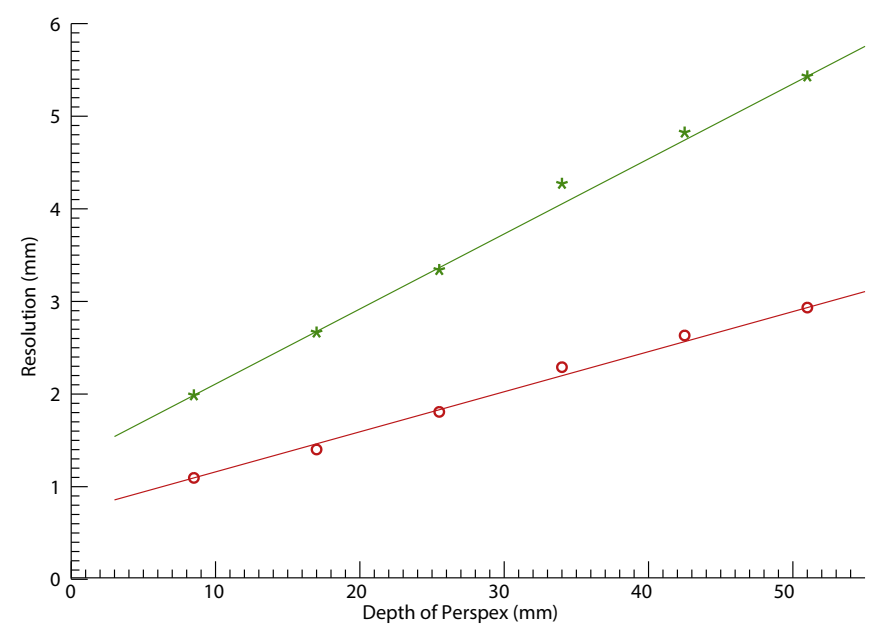

Figure 4. Plot of FWHM resolution (red dots) and FWTM resolution (green crosses) with lines of best fit for a range of Perspex thicknesses. Measurements are taken from images of a $1 \mathrm{~mm}$ diameter $125 \mathrm{~mm}$ long capillary tube containing $40 \mathrm{MBq}$ of ${ }^{99 \mathrm{~m}} \mathrm{Tc}$. The distance from the tube to the $0.5 \mathrm{~mm}$ diameter pinhole collimator is equal to the thickness of Perspex used. Resolution has been corrected for differences in pinhole magnification at different distances. (For interpretation of the references to colour in this figure legend, the reader is referred to the web version of this article.) each pixel, using the five nearest pixels in a row and a column, across an entire image [3]. A histogram can show the spread of these values and its width $(\delta \Omega)$ is the value suggested for reporting, which may be calculated directly using Equation (3),

$\delta \Omega=\frac{\left[\sum_{a=1}^{p} D U(a)^{2} n(a)\right]^{\frac{1}{2}}}{\sum_{a=1}^{p} n(a)}$

where $n(a)$ is the number of occasions on which a differential uniformity of $\operatorname{DU}(a)$ is found and $p$ is the number of data classes in the histogram.

Spatial uniformity was measured with a number of flood images, obtained using a $3 \mathrm{~mm}$ diameter $25 \mathrm{MBq}{ }^{99 \mathrm{~m}} \mathrm{Tc}$ filled source at a distance of $250 \mathrm{~mm}$ from the uncollimated detector face. At this distance the phantom behaved as a flood source, illuminating the detector evenly. Approximately 12,000 counts were collected. Flood images illustrate the need for flat field correction; Fig. 5 shows a flood image before and after correction for flat field effects.

Pixel photo-response non-uniformity was calculated for a CCD of the same model without a coupled scintillator. Although exact values of non-uniformity will vary with different CCDs, they are expected to be of a similar order. Output signal was plotted against exposure time for each individual pixel in a uniformly illuminated image and the proportionality constants (gradient of exposuresignal curve) compared. The proportionality constant varied by $4.5 \%$ within individual rows on the $\mathrm{CCD}$.

A larger non-uniformity effect is introduced by variability in thermal effects across the CCD - partly due to a temperature gradient and partly due to longer readout times for some pixels. This effect is seen in Fig. 5 with the top of the image showing a trend towards more counts than the bottom. Over the entire CCD the proportionality constant for each pixel varied by nearly $35 \%$ in total and showed a linear increase $\left(R^{2}=0.9982\right)$ from the bottom to the top of the CCD image. The magnitude of this effect will depend on the operation temperature of the $\operatorname{CCD}\left(-5{ }^{\circ} \mathrm{C}\right.$ for this test) although trends should remain consistent.

Non-uniformity may also be introduced by defects in the scintillator crystal and the coupling between it and the EMCCD. The structures seen in Fig. 5 are caused by thickness variations in the coupling optical grease and were not present before this method of coupling was introduced. These effects combined give an integral uniformity of $68 \%$ for the pre-flat-field corrected image in Fig. 5.

In clinical images these intrinsic non-uniformity effects will be combined with the non-uniformity of the pinhole collimator. Based on the camera geometry this would be expected to vary by approximately $20 \%$ [8] across the CCD due to changes in sensitivity with angle. This should be corrected for in clinical imaging by using a flood mask taken with the pinhole in place. Typically LFOV cameras report intrinsic spatial uniformity, without a collimator in place. These parameters for the CGC are displayed in Table 3.

The differential uniformity was lower than the integral uniformity, suggesting there is systematic variation across the detector. Alternatively a single very high or very low count pixel could have disproportionally affected the calculated result.

Table 2

CGC intrinsic spatial linearity measurements.

\begin{tabular}{lc}
\hline & Measurement $(\mu \mathrm{m})$ \\
\hline Mean deviation & 117 \\
Minimum deviation & 0 \\
Maximal deviation (absolute linearity) & 429 \\
Differential linearity & 93 \\
\hline
\end{tabular}



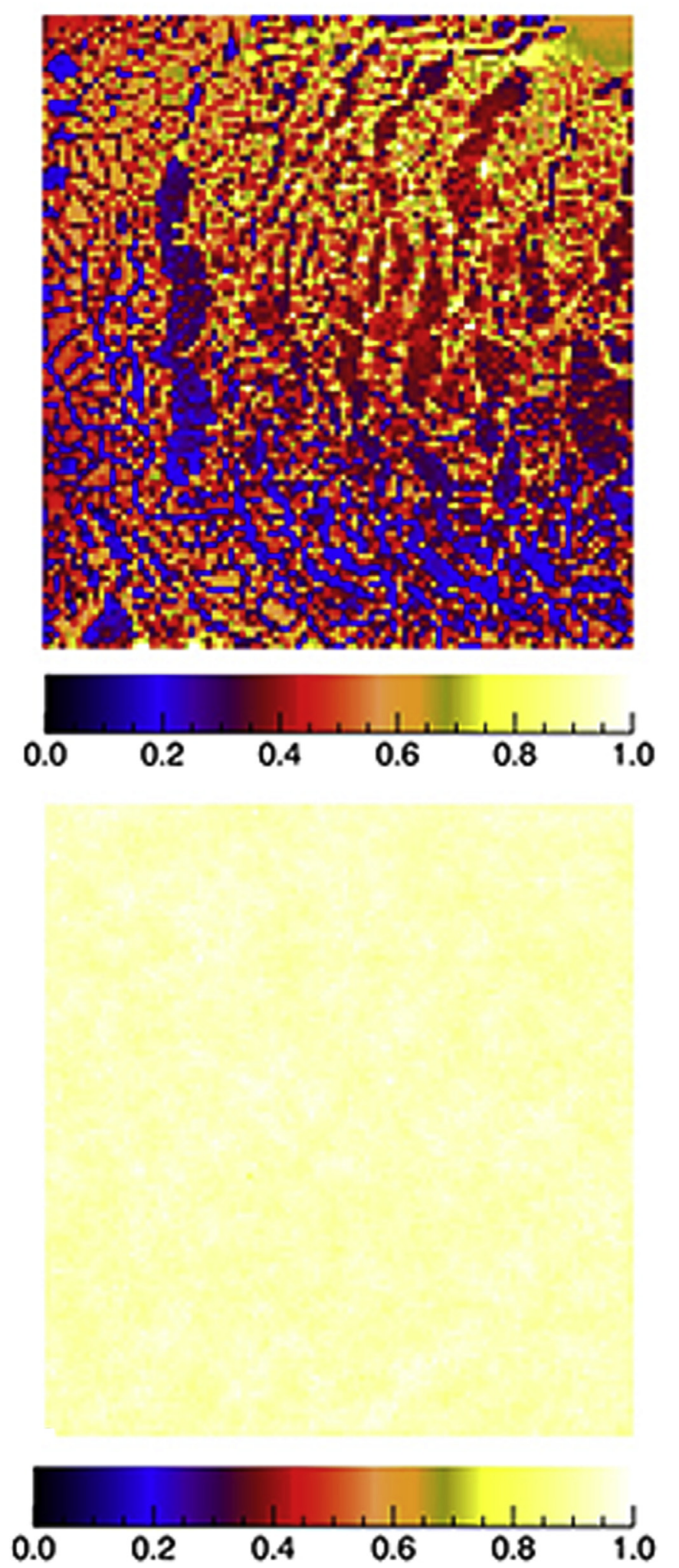

Figure 5. Top: Raw flood image normalised to maximum value. Bottom: Flat field corrected flood image, normalised to the corrected maximum value. Flood images were taken with a $3 \mathrm{~mm}$ diameter, $25 \mathrm{MBq}{ }^{99 \mathrm{~m}} \mathrm{Tc}$ filled source at a distance of $250 \mathrm{~mm}$ away from the uncollimated detector face. The flat field image used for correction was taken using the same set up, approximately $2 \mathrm{~h}$ prior to the image shown here. The dark image used in correction was also taken on the same day.

\section{Sensitivity}

Sensitivity is a measure of the proportion of incident photons which are detected. This is stated both without a collimator (intrinsic) and with a collimator in place (system). As this requires a quantitative description of detected counts, uniformity corrections were not applied.

To measure the intrinsic sensitivity, a $3 \mathrm{~mm}$ point source with an activity of $21 \mathrm{MBq}$ of ${ }^{99 \mathrm{~m}} \mathrm{Tc}$ was positioned $350 \mathrm{~mm}$ from the camera face to produce flood images. The expected incident counts on the detector were calculated based on the source-detector height, detector size and the activity of the source. Figure 6 shows the sensitivity of the detector, in terms of incident counts, for a range of Perspex thicknesses.

An exponential curve was fitted (Adj. $\left.R^{2}=0.993\right)$ to the data shown in Fig. 6; this fitted curve indicates a linear attenuation coefficient of the scattering material for photons of this energy of $0.137 \mathrm{~cm}^{-1}$. The per incident count rates, although useful, would not be comparing like with like when it comes to IPEM standards which require the cps/MBq of a point source. The data in Fig. 6 was extrapolated to give a sensitivity of approximately $62,300 \mathrm{cps} / \mathrm{MBq}$ (incident) at the detector surface. This result for incident counts can be used to calculate sensitivity in terms of the expected count rate for point sources at a range of heights. Results of this, assuming no intervening scattering medium, can be seen in Table 4. In practice, camera design limits the closest approach to the detector at $10 \mathrm{~mm}$, $\sim 2860 \mathrm{cps} / \mathrm{MBq}$ would therefore be a comparable value to LFOV measurements.

To calculate the extrinsic resolution of the system the sensitivity of the pinhole collimator must also be taken into account. Pinhole collimators have a well-established height-dependent sensitivity that can be calculated from Equation (4) [8].

$$
\begin{aligned}
S= & \frac{d^{2} \sin ^{3} \theta}{16 h^{2}}+\left[\frac{\sin ^{5} \theta \tan ^{2} \frac{\alpha}{2}}{8 h^{2} \mu^{2}}\right] \times\left[1-\frac{\cot ^{2} \theta}{\tan ^{2} \frac{\alpha}{2}}\right]^{\frac{1}{2}}\left[1-\frac{\cot ^{2} \theta}{\tan ^{2} \frac{\alpha}{2}}\right. \\
& \left.+\frac{\mu d}{\sin \theta \tan \frac{\alpha}{2}}\right]
\end{aligned}
$$

where $d$ is the diameter of the pinhole, $\theta$ is the angle of the source to the pinhole, $h$ is the distance from source to pinhole centre, $\alpha$ the acceptance angle of the pinhole and $\mu$ the linear attenuation of the pinhole material for the energy of photons used.

The tungsten collimator used for characterisation of the CGC has $d=0.5 \mathrm{~mm}, \alpha=60^{\circ}$ and $\mu=32.17 \mathrm{~cm}^{-1}$ for gamma rays of $141 \mathrm{keV}$. The collimator is $6 \mathrm{~mm}$ thick in total with a centrally positioned pinhole. A source placed on the collimator surface would therefore be a distance of $3 \mathrm{~mm}$ from the pinhole.

Collimator sensitivity can be calculated using Equation (4). At a height of $3 \mathrm{~mm}$ with a source-pinhole angle of $90^{\circ}$ (i.e. the source placed directly on top of the pinhole collimator) this gives us a pinhole sensitivity of $3430 \mathrm{cps} / \mathrm{MBq}$. Combining this with intrinsic sensitivity, gives us an extrinsic sensitivity on the camera face of $214 \mathrm{cps} / \mathrm{MBq}$.

Table 3

CGC spatial uniformity measurements.

\begin{tabular}{ll}
\hline & Measurement (\%) \\
\hline Coefficient of variation & 1.58 \\
Integral uniformity & 8.50 \\
Mean differential uniformity & 1.32 \\
Spread of differential uniformity & 0.60 \\
\hline
\end{tabular}




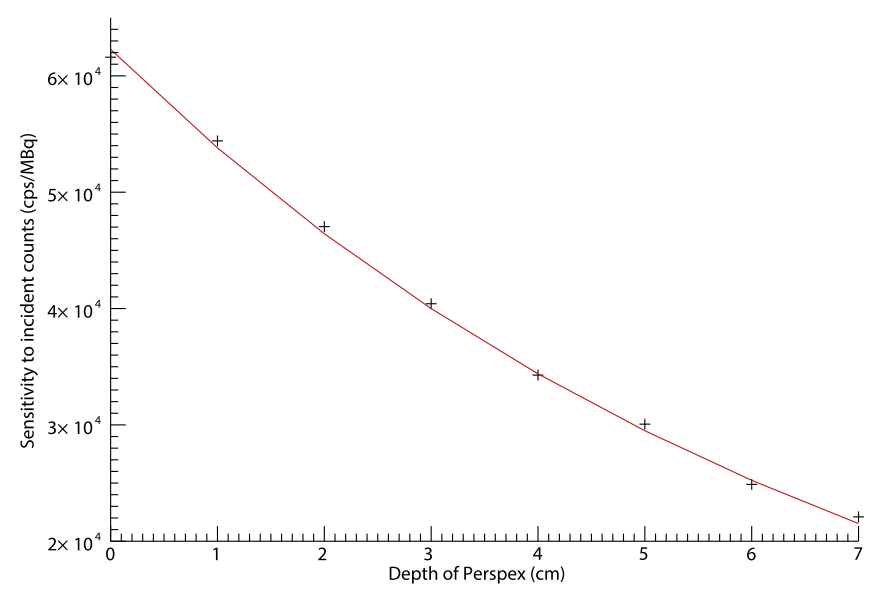

Figure 6. Sensitivity of uncollimated CGC for a range of Perspex distances. Flood images were taken with a $3 \mathrm{~mm}$ diameter, $21 \mathrm{MBq}{ }^{99 \mathrm{~m}} \mathrm{Tc}$ filled source at a distance of $350 \mathrm{~mm}$ away from the uncollimated detector face. Source distanced remained fixed with varying depths of Perspex placed between the source and the detector. Total counts were taken from images with no flat field correction applied and compared to incident counts calculated based on source activity and distance to detector using solid angle formulae.

\section{Count rate capability}

The count rate capability of the CGC is affected by two factors, firstly the count rate capability of the detector itself - the saturation charge level - and secondly the counts per frame required to resolve each individual event. This second measure of count rate capability is the more practical for comparison with other cameras and is largely dependent on the frame rate determined by detector readout (currently $10 \mathrm{~Hz}$ for the $\mathrm{CGC}$ ).

Figure 7 shows the count rate capability data when using the blob detection algorithm, as would occur in practice. This was found using a $20 \mathrm{MBq}{ }^{99 \mathrm{~m}} \mathrm{Tc}$ source at distance of $350 \mathrm{~mm}$ from the detector over a period of $24 \mathrm{~h}$.

A straight line was fitted to the proportional section of the curve $\left(R^{2}=0.9996\right)$ indicating that the detector behaves linearly until at least 1200 cps incident. Testing on a similar detector has shown measured values began to vary by more than $20 \%$ from this fitted line at $2.2 \mathrm{kBq}$ incident. This is due to the nature of the algorithm which requires distinct light splashes for fitting. Where more than one light splash overlaps, the algorithm is unable to resolve these as separate events and this leads to the 'saturation'. The maximum observed count rate of 30/s corresponds to approximately 3 events per frame.

As in Table 4, the count rate capability may be converted to point source values. This would relate to a point source of $27 \mathrm{kBq}$ at a distance of $10 \mathrm{~mm}$ from the uncollimated detector.

\section{Energy resolution}

An $18 \mathrm{MBq}{ }^{99 \mathrm{~m}} \mathrm{Tc}$ source was placed at a distance of $45 \mathrm{~mm}$ from the camera and the energy spectrum recorded using the blob

Table 4

CGC intrinsic sensitivity for point sources.

\begin{tabular}{lc}
\hline Height $(\mathrm{mm})$ & Intrinsic sensitivity $(\mathrm{cps} / \mathrm{MBq})$ \\
\hline 10 & 2860 \\
20 & 800 \\
50 & 130 \\
100 & 30 \\
200 & 20 \\
\hline
\end{tabular}

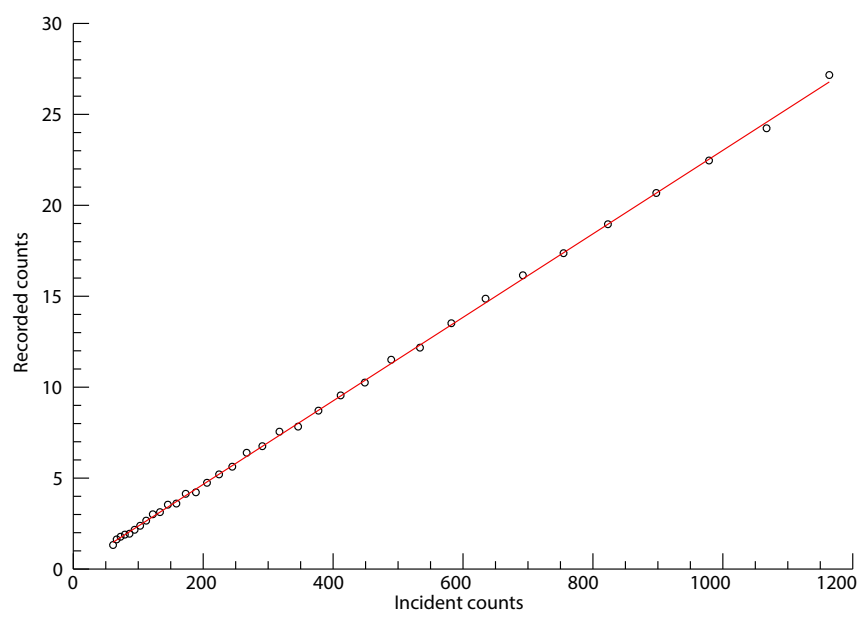

Figure 7. Frame rate limited count rate capability for CGC detector. Images were taken with a $3 \mathrm{~mm}$ diameter, $20 \mathrm{MBq}{ }^{99 \mathrm{~m}} \mathrm{Tc}$ filled source at a distance of $350 \mathrm{~mm}$ away from the uncollimated detector face. Incident counts calculated using solid angle formula and activity of source with decay over time.

detection algorithm. Similarly, a ${ }^{109} \mathrm{Cd}$ source $(4 \mathrm{MBq})$ at a height of $5 \mathrm{~mm}$ was used to produce a second spectrum. Flat field correction was not applied for these tests. These are compared in Fig. $8 .{ }^{109} \mathrm{Cd}$ produces photons of 22.16 and $24.94 \mathrm{keV}$; photons of both energies contribute to the single peak in Fig. 8 at $\sim 23 \mathrm{keV}$. As FWHM of the ${ }^{109} \mathrm{Cd}$ peak is $20.4 \mathrm{keV}$ it is not expected the peaks of the ${ }^{109} \mathrm{Cd}$ spectrum will be resolvable.

The FWHM of the ${ }^{99 \mathrm{~m}} \mathrm{Tc}$ peak, at $141 \mathrm{keV}$, is $82.4 \mathrm{keV}$; an energy resolution of $58 \%$. At the lower energy end of the ${ }^{99 m}$ Tc spectrum a large noise tail could be seen, along with fluorescence. The peak at approximately $30 \mathrm{keV}$ can be identified as the overlaid fluorescence peaks from the scintillator (Cs-K $31 \mathrm{keV}, \mathrm{I}-\mathrm{K} 28 \mathrm{keV}$ ).

\section{Discussion}

The CGC was fully characterised with its performance quantified for spatial resolution, spatial linearity, image uniformity, sensitivity, count rate capability and energy resolution. These measurements are collated in Table 5.

It is now possible to compare both LFOV cameras currently in clinical use and SFOV cameras being developed for imaging in the future for all measurements given in Table 5. For systems using photon multiplier tubes (PMTs), values are usually given for both the Usable Field of View (UFOV) and the Central Field of View (CFOV) to account for variation towards the edges of the detector matrix. In Table 5, CFOV measurements have been used exclusively when provided. A $X$ indicates information was not available. All distance dependent values were given at $13 \mathrm{~mm}$ above the collimator for the CGC - the point with no magnifying effect. When multiple values for resolution were present in literature, the value at the distance closest to $13 \mathrm{~mm}$ was chosen. Where multiple collimator values were found in the literature, the collimator that gave the best performance for the parameter in question was chosen.

The intrinsic spatial resolution of the CGC compares favourably to most other systems. This is mainly due to the use of a CCD detector as opposed to PMTs - CCDs have naturally better resolution due to the smaller pixel size. A camera that uses a similar detection system, the UGC, reported a far better intrinsic resolution (60$90 \mu \mathrm{m})$ despite having a similar design. However, the UGC resolution was measured at the crystal face, whereas the CGC resolution was measured with the camera assembled for clinical imaging and so approach to the crystal was limited to $10 \mathrm{~mm}$. 


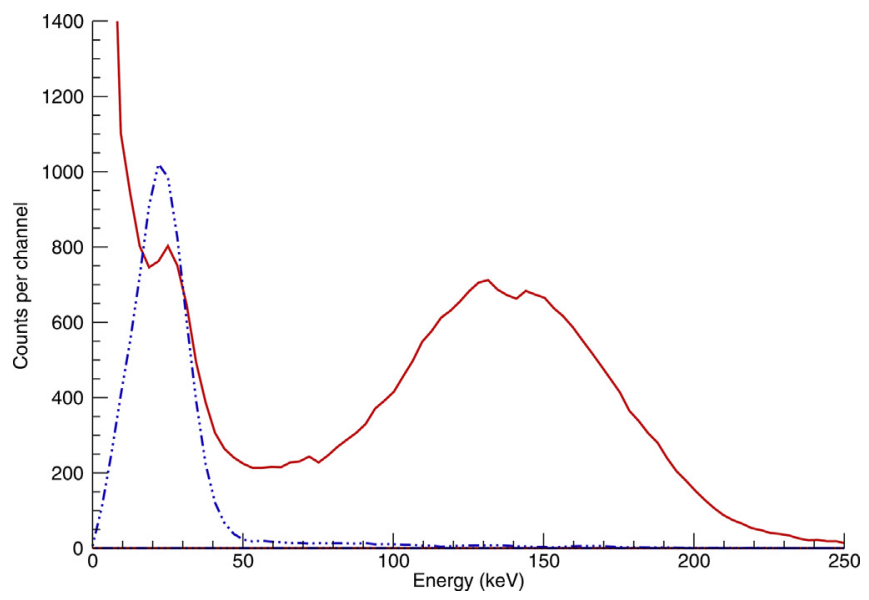

Figure 8. Spectral data for ${ }^{99 \mathrm{~m}} \mathrm{Tc}$ (solid red) and ${ }^{109} \mathrm{Cd}$ (dotted blue) sources. The ${ }^{99 \mathrm{~m}} \mathrm{Tc}$ source had an activity of $18 \mathrm{MBq}$ and was placed $45 \mathrm{~mm}$ from the collimator. The ${ }^{109} \mathrm{Cd}$ source had an activity of $4 \mathrm{MBq}$ and was placed $5 \mathrm{~mm}$ from the collimator. Energy in arbitrary digital units for each incident photon was calculated through the blob detection algorithm. The ${ }^{109} \mathrm{Cd}$ peak was used to calibrate the energy scale to $\mathrm{eV}$. (For interpretation of the references to colour in this figure legend, the reader is referred to the web version of this article.)

In practice, the intrinsic resolution of a detector is small compared to the system resolution and acts to reduce contrast in an image taken with a collimator in place. For this reason, a comparison of system spatial resolution provides a better indication of how a camera will behave in practice.

System spatial resolution also compared favourably to other systems, with a FWHM the second lowest of all the discussed cameras. It is worth noting that this is the resolution at the nonmagnifying point and that resolution will degrade at greater values. Resolution will also increase at distance for other systems; the POCI camera has a resolution varying from $3.2 \mathrm{~mm}$ FWHM at the collimator face to $7.5 \mathrm{~mm}$ through $50 \mathrm{~mm}$ of a scattering medium [9]. For comparison, the CGC has a calculated resolution of $2.9 \mathrm{~mm}$ through $50 \mathrm{~mm}$ of a scattering medium, still considerably less. The difference in proportional variation is simply due to the different behaviour of pinhole (CGC) and parallel hole (POCI) collimation.

The CGC detector has a spatial linearity well within the range expected for clinical systems. The CGC detector is significantly smaller than that of LFOV systems so it is to be expected that linearity measurements, particularly absolute linearity, are lower than these systems.

Integral uniformity for the CGC is higher than that of other cameras, with only one other camera being less uniform. Integral uniformity, however, is a less robust method of quantification than the coefficient of variation (1.58\% for the CGC) which is the IPEM suggested parameter - it is excluded from Table 5 as it is not typically given for other systems. The SSGC does provide the coefficient of variation for its integral uniformity as $4.5 \%$ and the CGC coefficient of variation compares favourably with this [10]. The comparatively high integral uniformity in Table 5 is more likely an issue with the method of calculation of integral uniformity, not the integral uniformity itself.

The spread of differential uniformity shows better performance than other systems. The CGC has the best spatial uniformity measured by this parameter. The uniformity of the CGC will also improve once the method of coupling the scintillator to the CCD has been perfected. Figure 5 shows the variation caused by the current method and this variation should be reduced in future iterations of the CGC.
At the time of writing, count rate capability of the CGC was a factor of 100 lower than other systems, although at this level of activity the full count rate capability of the detector has not been reached. It is expected that a camera with a larger detecting area will have a higher count rate capability. Considering count rate per $\mathrm{cm}^{2}$ of detector, the CGC would have a capability of $>1.9 \mathrm{kBq} / \mathrm{cm}^{2}$, compared to the Solomobile, with a capability of $0.5 \mathrm{kBq} / \mathrm{cm}^{2}$. When size effects are accounted for in this way the CGC's count rate capability is superior to other systems.

Count rate capability of the CGC is limited by the ability to resolve different light splashes on the detector. A similar set up was seen to saturate at approximately 3 events per frame. The current CGC runs at $10 \mathrm{~Hz}$ (30 resolvable events per second). A CCD of the same type has achieved a frame rate of $180 \mathrm{~Hz}$ [23]. Were the CGC able to run at rates of even half this, it would be able to resolve approximately 270 events per second. Assuming the relationship of incident to observed counts remains consistent over this range, the CGC should be able to achieve count rate capabilities in the range of approximately $20 \mathrm{kBq}$. This would represent a significant improvement, bringing the CGC to within a factor of 10 of count rate capability for clinical systems, even without corrections for detector area. This is a factor that will be improved upon in further iterations of the electronic systems used by the CGC.

Sensitivity was good compared to other cameras considering that the CGC uses a single $0.5 \mathrm{~mm}$ diameter pinhole as opposed to the more typical parallel hole collimator. Sensitivity is a hugely important factor in clinical imaging, where the goal is always to expose the patient to as little activity as possible. The CGC shows comparable sensitivity to parallel hole systems alongside an improvement in spatial resolution, parameters which usually require a trade-off. The use of the pinhole, however, will mean that the sensitivity of the system will drop off faster at distance than parallel hole systems which is a factor that would need to be taken into account in clinical studies.

The energy resolution of the CGC was considered to be poor compared to the other cameras looked at here. Theoretically, the energy resolution of detector is limited by photoelectron statistics, themselves limited by the quantum efficiency of the detector, the energy conversion efficiency of the scintillator, and the light collection efficiency in the coupling between the scintillator and detector, along with impurities and imperfections in the scintillating crystal. Assuming a 50\% light collection efficiency, perfect quantum efficiency and no crystalline imperfections, we can estimate the maximum energy resolution of the CGC detector to be $\sim 20 \%$ at $141 \mathrm{keV}$, which is more in line with other SFOV scintillator cameras investigated e.g. $24 \%$ for the UGC. Further iterations of the blob detection algorithm used with the CGC will include depth of interaction calculations which have been shown to improve energy resolution measurements in similar systems along with having additional benefits [24]. Improvements in coupling between the scintillator and CCD will also confer a large benefit here. Fluorescence may also be the cause of some the spread in the ${ }^{99 \mathrm{~m}} \mathrm{Tc}$ peak, in this case fluorescence within the tungsten collimator and lead shielding (59-85 keV). At the higher end, spreading may also be caused by overlapping light splashes being analysed as a single event. Both possibilities are currently under investigation through simulation experiments. Semiconductor detectors report energy resolutions of less than $10 \%$ it is expected that these cameras will perform better than scintillator cameras due to the direct conversion of gamma energy to electronic signal [1].

Overall the CGC performance characteristics compare well to those of other cameras in use and in development. The overall performance is not limited by the design of the camera and this report outlines steps that will be taken to further improve several parameters in future developmental models. 
Table 5

Comparison of performance characteristics for a range of gamma cameras.

\begin{tabular}{|c|c|c|c|c|c|c|c|c|c|c|}
\hline & & \multirow[t]{2}{*}{$\mathrm{CGC}^{\mathrm{a}}$} & \multicolumn{4}{|c|}{ LFOV scintillator/PMT detectors } & \multicolumn{4}{|c|}{ SFOV scintillator/PSPMT detectors } \\
\hline & & & $\begin{array}{l}\text { Nucline }^{\mathrm{TM}} \\
\text { Cardio- }^{\mathrm{b}}[12]\end{array}$ & $\begin{array}{l}\text { Solomobile }^{\mathrm{b}} \\
\text { [11] }\end{array}$ & \multicolumn{2}{|c|}{$\begin{array}{l}\text { Infinia }^{\mathrm{TM}} \\
\text { Hawkeye }^{\mathrm{TM}} 4 \text { [13] }\end{array}$} & $\begin{array}{l}\text { Olcott } \\
\text { et al. }{ }^{\text {b }}[14]\end{array}$ & $\begin{array}{l}\text { Guardian2 } 2^{\mathrm{a}} \\
\text { [15] }\end{array}$ & $\begin{array}{l}\text { Trotta } \\
\text { et al. }{ }^{\text {a }}[16]\end{array}$ & $\begin{array}{l}\text { MONICA }^{\mathrm{b}} \\
{[17]}\end{array}$ \\
\hline \multirow{4}{*}{$\begin{array}{l}\text { Field of view } \\
\text { Intrinsic spatial } \\
\quad \text { resolution }\end{array}$} & Nominal (mm) & $40 \times 40$ & $370 \times 210$ & $210 \varnothing$ & \multicolumn{2}{|l|}{$540 \times 400$} & $50 \times 50$ & $44 \times 44$ & & $49 \times 92$ \\
\hline & FWHM (mm) & 0.63 & 2.8 & 2.7 & \multicolumn{2}{|l|}{3.8} & 1.8 & $\mathrm{X}$ & $\mathrm{X}$ & $\mathrm{X}$ \\
\hline & FWTM (mm) & 1.06 & 5.6 & 7.6 & \multicolumn{2}{|l|}{7.1} & $\mathrm{X}$ & $\mathrm{X}$ & $\mathrm{X}$ & $\mathrm{X}$ \\
\hline & MTF $10 \%(\mathrm{~mm})$ & 0.54 & $\mathrm{x}$ & $\mathrm{X}$ & $\mathrm{X}$ & & $\mathrm{X}$ & $\mathrm{X}$ & $\mathrm{X}$ & $\mathrm{X}$ \\
\hline System spatial & FWHM $(\mathrm{mm})$ & 1.28 & 7.1 & 7.8 & 7.4 & & $\mathrm{X}$ & 2.5 & $\mathrm{X}$ & 2.2 \\
\hline resolution & FWTM (mm) & 2.35 & $\mathrm{X}$ & $\mathrm{X}$ & $\mathrm{X}$ & & $\mathrm{X}$ & $\mathrm{X}$ & $\mathrm{X}$ & $\mathrm{X}$ \\
\hline Spatial linearity & Absolute (mm) & 0.12 & 0.38 & 0.5 & 0.5 & & $\mathrm{X}$ & 0.4 & $\mathrm{X}$ & $\mathrm{X}$ \\
\hline & Differential (mm) & 0.09 & 0.18 & 0.2 & 0.1 & & $\mathrm{X}$ & $\mathrm{X}$ & $\mathrm{X}$ & $\mathrm{X}$ \\
\hline Intrinsic spatial & Integral uniformity (\%) & 8.5 & 2.4 & 2.5 & 3.0 & & $\mathrm{X}$ & 8.8 & $\mathrm{X}$ & $<3$ \\
\hline uniformity & $\begin{array}{l}\text { Spread of differential } \\
\text { uniformity (\%) }\end{array}$ & 0.60 & 1.9 & 1.7 & 2.1 & & $\mathrm{X}$ & 4.0 & $\mathrm{X}$ & $\mathrm{X}$ \\
\hline Count-rate capability & y $20 \%$ Expected value $(\mathrm{kBq}$ & q) $>1.2$ & 200 & 180 & 300 & & $\mathrm{X}$ & $\mathrm{X}$ & $\mathrm{X}$ & $>275$ \\
\hline & Maximum counts $(\mathrm{kBq})$ & $>1.2$ & 220 & 250 & 370 & & $\mathrm{X}$ & $\mathrm{X}$ & $\mathrm{X}$ & $>275$ \\
\hline Sensitivity & Intrinsic (cps/MBq) & 2860 & $\mathrm{X}$ & $\mathrm{X}$ & $\mathrm{X}$ & & $\mathrm{X}$ & $\mathrm{X}$ & $\mathrm{X}$ & $\mathrm{X}$ \\
\hline & Extrinsic (cps/MBq) & 214 & 144 & 122 & 144 & & 135 & 204 & 211 & 149 \\
\hline Energy resolution & FWHM at $141 \mathrm{keV}(\%)$ & 58 & 9.7 & 9.4 & 9.8 & & 12.1 & 20 & 19.1 & 10.8 \\
\hline & & & Scintillator/photod & diode detectors & Scintillator & $C D d$ & tectors & Semiconductc & tor detectors & \\
\hline & & $\overline{\mathrm{CGC}^{\mathrm{a}}}$ & $\mathrm{POCI}^{\mathrm{C}}[9]$ & Ergo $^{a}[18]$ & $\mathrm{UGC}^{\mathrm{a}}$ [19] & & $\begin{array}{l}\text { sarkar } \\
\text { l. }{ }^{\mathrm{a}}[20]\end{array}$ & $\begin{array}{l}\text { eZ SCOPE }^{\mathrm{d}} \\
{[21]}\end{array}$ & $\operatorname{SSGC}^{\mathrm{e}}[10]$ & $\begin{array}{l}\text { MediPROBE }^{\mathrm{e}} \\
{[22]}\end{array}$ \\
\hline Field of view & Nominal (mm) & $40 \times 40$ & $40 \varnothing$ & $396 \times 311$ & $24 \times 18$ & & $\times 100$ & $32 \times 32$ & $44.8 \times 44.8$ & $70.4 \times 70.4$ \\
\hline Intrinsic spatial & FWHM (mm) & 0.63 & 2.3 & $\mathrm{X}$ & $0.06-0.09$ & 0.6 & & $\mathrm{X}$ & $\mathrm{X}$ & $\mathrm{X}$ \\
\hline resolution & FWTM (mm) & 1.06 & $\mathrm{X}$ & $\mathrm{X}$ & $\mathrm{X}$ & $\mathrm{X}$ & & $\mathrm{X}$ & $\mathrm{X}$ & $\mathrm{X}$ \\
\hline & MTF $10 \%(\mathrm{~mm})$ & 0.54 & $\mathrm{X}$ & $\mathrm{X}$ & $\mathrm{X}$ & $\mathrm{X}$ & & $\mathrm{X}$ & $\mathrm{X}$ & $\mathrm{X}$ \\
\hline System spatial & FWHM (mm) & 1.28 & 3.2 & 3.0 & $\mathrm{X}$ & 1.2 & & 2.2 & 1.56 & 1.8 \\
\hline resolution & FWTM (mm) & 2.35 & $\mathrm{X}$ & $\mathrm{X}$ & $\mathrm{X}$ & $\mathrm{X}$ & & $\mathrm{X}$ & $\mathrm{X}$ & $\mathrm{X}$ \\
\hline Spatial linearity & Absolute (mm) & 0.12 & $\mathrm{X}$ & $\mathrm{X}$ & $\mathrm{X}$ & $\mathrm{X}$ & & $\mathrm{X}$ & $\mathrm{X}$ & $\mathrm{X}$ \\
\hline & Differential (mm) & 0.09 & $\mathrm{X}$ & $\mathrm{X}$ & $\mathrm{X}$ & $\mathrm{X}$ & & $\mathrm{X}$ & $\mathrm{X}$ & $\mathrm{X}$ \\
\hline Intrinsic spatial & Integral uniformity (\%) & 8.5 & $\mathrm{X}$ & $<5$ & $\mathrm{X}$ & $\mathrm{X}$ & & 1.6 & 4.5 & $\mathrm{X}$ \\
\hline uniformity & $\begin{array}{l}\text { Spread of differential } \\
\text { uniformity (\%) }\end{array}$ & 0.60 & $\mathrm{X}$ & $<3$ & $\mathrm{X}$ & $\mathrm{X}$ & & 1.3 & $\mathrm{X}$ & $\mathrm{X}$ \\
\hline Count-rate & $20 \%$ Expected value ( $\mathrm{kBq})$ & $>1.2$ & $\mathrm{X}$ & $>20,000$ & $\mathrm{X}$ & $\mathrm{X}$ & & $>180$ & $\mathrm{X}$ & $\mathrm{X}$ \\
\hline capability & Maximum counts $(\mathrm{kBq})$ & $>1.2$ & $\mathrm{X}$ & $\mathrm{X}$ & $\mathrm{X}$ & $\mathrm{X}$ & & $>180$ & $\mathrm{X}$ & $\mathrm{X}$ \\
\hline Sensitivity & Intrinsic (cps/MBq) & 2860 & $\mathrm{X}$ & $\mathrm{X}$ & $\mathrm{X}$ & $\mathrm{X}$ & & $\mathrm{X}$ & $\mathrm{X}$ & $\mathrm{X}$ \\
\hline & Extrinsic (cps/MBq) & 214 & 290 & 112 & $\mathrm{X}$ & $\mathrm{X}$ & & 477 & 300 & $>100$ \\
\hline Energy resolution & FWHM at $141 \mathrm{keV}(\%)$ & 58 & 32 & 7.4 & 24 & $\mathrm{X}$ & & 8.6 & $4.2-7.8$ & $\mathrm{X}$ \\
\hline
\end{tabular}

${ }^{\text {a }} \mathrm{CsI}(\mathrm{Tl})$ scintillator.

b $\mathrm{NaI}(\mathrm{Tl})$ scintillator.

c $\mathrm{CsI}(\mathrm{Na})$ scintillator.

d CdZnTe semiconductor.

e CdTe semiconductor.

\section{Conclusion}

The procedures described in this communication were successful in translating LFOV standards to methods applicable to the CGC. A more generalised set of protocols that will be applicable to all SFOV cameras is in development and will be published at a later date. SFOV cameras are often designed for specific purposes such as sentinel lymph node biopsy, parathyroid gland surgery and radioimmunoguided surgery [1]. In addition to the transfer of LFOV protocols, standardised tests should be established for the specific procedures where a SFOV camera may be used. This will be discussed in a future publication.

Our results show that the CGC has the potential to provide highresolution clinical images with a good level of uniformity and a sensitivity comparable to other systems over small distances. We have also shown areas for improvement in future iterations which, based on theoretical calculations, will bring the CGC in line with other systems in terms of count rate capability and energy resolution.

\section{Conflicts of interest}

SLB and BSB declare that they have no conflicts of interest. Both JEL and ACP are Directors of a Universities of Leicester and Nottingham spin out company called Gamma Technologies.

\section{Acknowledgements}

The authors would like to thank David Bassford of the University of Leicester and Elaine Blackshaw of Nottingham University Hospitals NHS Trust, for technical assistance in collecting the results used for this report and Adam Bark and Oliver Blake, both of the University of Leicester, for their assistance in developing the acquisition and blob detection software used. This work was supported by a Science and Technologies Facilities Council studentship and an STFC CLASP grant (ST/I00327/4).

\section{References}

[1] Tsuchimochi M, Hayama K. Intraoperative gamma cameras for radioguided surgery: technical characteristics, performance parameters, and clinical applications. Phys Med 2013;29(2):126-38.

[2] National Electrical Manufacturers Association, NU1-2007 1300 North 17th Street, Suite 1752, Rosslyn, Virginia 22209, USA.

[3] IPEM. In: Bolster A, editor. Quality control of gamma camera systems. York: Institute of Physics and Engineering in Medicine; 2003. Report No. 86.

[4] Lees JE, Bassford DJ, Blake OE, Blackshaw PE, Perkins AC. A high resolution Small Field of View (SFOV) gamma camera: a columnar scintillator coated CCD imager for medical applications. J Instrum 2011;6(12):C12033.

[5] CCD97-00-Back illuminated 2-phase IMO series peltier pack electron multiplying CCD sensor. Chelmsford, Essex, CM1 2QU, England: E2V Technologies Limited; 2011. A1A-CCD97.

[6] Goulet P. Resolution of pinhole collimators used with gamma cameras. Med Phys (Lancaster) 1980;7(5):571. 
[7] Bugby SL, Lees JE, Perkins AC. Modelling image profiles produced with a small field of view gamma camera with a single pinhole collimator. J Instrum 2012;7(11):P11025

[8] Metzler SD, Bowsher JE, Smith MF, Jaszczak RJ. Analytic determination of pinhole collimator sensitivity with penetration. Med Imaging IEEE Trans 2001;20(8):730-41.

[9] Pitre S, Menard L, Ricard M, Solal M, Garbay JR, Charon Y. A hand-held imaging probe for radio-guided surgery: physical performance and preliminary clinical experience. Eur J Nucl Med Mol Imaging 2003;30(3):339-43.

[10] Tsuchimochi M, Sakahara H, Hayama K, Funaki M, Ohno R, Shirahata T, et al. A prototype small CdTe gamma camera for radioguided surgery and other imaging applications. Eur J Nucl Med Mol Imaging 2003;30(12):1605-14.

[11] Product data SoloMobile. DK-2970 Hørsholm, Denmark: D.N.v.E. DDD-Diagnostic; 2012

[12] Nucline cardio-C data sheet. Alsótőrökvész u. 14, Hungary: H.-B. Mediso Medical Imaging Systems; 2004.

[13] Infinia Hawkeye 4 product datasheet. WI, USA: M. GE Healthcare; 2006. 5195413-100.

[14] Olcott PD, Habte F, Foudray AM, Levin CS. Performance characterization of a miniature, high sensitivity gamma ray camera. Nucl Sci IEEE Trans 2007;54(5):1492-7.

[15] Ferretti A, Chondrogiannis S, Marcolongo A, Rubello D. Phantom study of a new hand-held gamma-imaging probe for radio-guided surgery. Nucl Med Commun 2013;34(1):86-90.
[16] Trotta C, Massari R, Palermo N, Scopinaro F, Soluri A. New high spatial resolution portable camera in medical imaging. Nucl Instrum Methods Phys Res Sect A Accel Spectrom Detect Assoc Equip 2007;577(3):604-10.

[17] Xi W, Seidel J, Kakareka JW, Pohida TJ, Milenic DE, Proffitt J, et al. MONICA: a compact, portable dual gamma camera system for mouse whole-body imaging. Nucl Med Biol 2010;37(3):245-53.

[18] Siman W, Kappadath SC. Performance characteristics of a new pixelated portable gamma camera. Med Phys 2012;39(6):3435-44.

[19] Beekman FJ, de Vree GA. Photon-counting versus an integrating CCD-based gamma camera: important consequences for spatial resolution. Phys Med Biol 2005;50(12):N109-19.

[20] Nagarkar VV, Shestakova I, Gaysinskiy V, Tipnis SV, Singh B, Barber W, et al. A CCD-based detector for SPECT. Nucl Sci IEEE Trans 2006;53(1):54-8.

[21] Abe A, Takahashi N, Lee J, Oka T, Shizukuishi K, Kikuchi T, et al. Performance evaluation of a hand-held, semiconductor (CdZnTe)-based gamma camera. Eur J Nucl Med Mol Imaging 2003:30(6):805-11.

[22] Russo P, Curion AS, Mettivier G, Esposito M, Aurilio M, Caraco C, et al. Evaluation of a CdTe semiconductor based compact gamma camera for sentinel lymph node imaging. Med Phys 2011;38(3):1547-60.

[23] Tulloch SM. Photon counting and fast photometry with L3 CCDs; 2004. p. 604-14.

[24] Korevaar MA, Heemskerk JW, Beekman FJ. A pinhole gamma camera with optical depth-of-interaction elimination. Phys Med Biol 2009;54(13): N267-72. 